Check for updates

Cite this: RSC Adv., 2019, 9, 14567

Received 27th February 2019

Accepted 28th April 2019

DOI: 10.1039/c9ra01507h

rsc.li/rsc-advances

\section{Detecting early stage structural changes in wild type, pathogenic and non-pathogenic prion variants using Markov state model $\uparrow$}

\begin{abstract}
Vinod Jani, Uddhavesh Sonavane and Rajendra Joshi (DD *
The conversion of prion protein from normal to scrapie followed by the aggregation and deposition of this scrapie form leads to various neurodegenerative diseases. A few studies carried out by researchers suggest that E219K prion mutant (glutamate to lysine mutation at residue position 219) is more stable than wild type protein. However a similar point mutation E200K (glutamate to lysine mutation at residue position 200) is pathogenic. In this study we have carried out detailed atomistic simulation of the wild type, pathogenic mutant E200K and E219K mutant which provides more stability. The aim of the study was to detect the early structural changes present in all the three variants which might be responsible for the stability or for their conversion from PrPC to PrPSC. MSM based analyses have been carried out to find out the differences between WT, E200K and E219K systems. Markov state model (MSM) analysis was able to predict the intermediate states which helped to understand the effect of same mutation at two different locations. The MSM analysis was able to show that the extra stability of E219K mutant may be a result of the increase in number of native contacts, strong salt bridges and less random motions. While pathogenicity of E200K mutant can be attributed to loss of some crucial salt-bridge interactions, increased random motions between helix 2 and helix 3 .
\end{abstract}

\section{Introduction}

The prion protein ( $\mathrm{PrP})$ is a membrane bound glycoprotein present in the neuronal cells of mammals. It is linked to the cell membrane via a glycophosphatidylinositol anchor present at its C-terminus. ${ }^{1,2}$ Structural changes in the prion protein are responsible for a group of neurodegenerative diseases known as prion diseases or transmissible spongiform encephalopathies (TSE). These diseases include scrapie in sheep, bovine spongiform encephalopathy (BSE) in cattle, and Creutzfeldt-Jakob disease (CJD), Gerstmann-StrausslerScheinker disease, fatal familial insomnia, and kuru in humans. $^{3-8}$ These structural changes in the prion protein may be attributed to the mutations occurring in the prion protein gene. These mutations may occur owing to an infection or inheritance. ${ }^{9,10}$

PrP is a glycoprotein of around 209 residues. Residues 23124 form the N-terminal region which is a highly disordered and unstructured region. Residues 126-231 form the C-

High Performance Computing-Medical \& Bioinformatics Applications Group, Centre for Development of Advanced Computing (C-DAC), Savitribai Phule Pune University Campus, Pune 411007, India.E-mail: rajendra@cdac.in

$\dagger$ Electronic supplementary information (ESI) available: Details of geometrical parameters used for carrying out feature based PCA, validating parameters for MSM and plots for geometrical parameters for MSM clusters. See DOI: 10.1039/c9ra01507h. terminal region, which is an ordered and folded domain. It comprises of three $\alpha$-helices namely helix H1, 144-154; helix H2, 173-195; helix H3, 200-226 and two short anti-parallel $\beta$ strands strand1(St1), 127-130; and strand2(St2), 162-165. The $\mathrm{H} 2$ and $\mathrm{H} 3$ are known to form a covalent bridge through by a disulfide bond between Cys179 and Cys214 (ref. 11-14). The normal prion protein or PrPC is monomeric, soluble, and protease-sensitive. Scrapie prion protein or PrPSc is highly insoluble and protease-resistant. ${ }^{\mathbf{1 0 , 1 5}}$ Both these forms differ in their structural content. The structure for the PrPSc is ill defined due to its insolubility and conformational heterogeneity. ${ }^{16}$ However it is known that the PrPSc is rich in $\beta$ content as compared to that of PrPC, which is rich in helical content. These structural differences are responsible for the difference in their physiochemical properties. The newly formed scrapie prion protein can induce nearby normal prion proteins to acquire a misfolded form. ${ }^{17}$ The scrapie prion proteins also have a tendency to aggregate and form dense amyloid fibrillary structure. It gets deposited within the neuronal cells and results in neurodegenerative disorders. It is also known that these structural changes comprise of $\alpha$ to $\beta$ refolding events and studying such events poses challenges in understanding their function. The structural changes in the prion protein are attributed to mutations hence studying dynamics of mutated protein can provide better understanding of these transition events. 
The structural changes that cause the conversion of PrPC to PrPSc and the mechanistic details of PrPSc self-propagation have not yet been understood completely. There are several studies which provide some understanding on fibrillation and oligomerization processes. ${ }^{18-21}$ However, an important concern is to identify a region or a group of residue sequences vulnerable to undergo conformational changes and act as a site for initial seeding and lead towards fibrillary growth. Earlier studies have suggested that the residues 125-165 comprising of St1-H1-St2 region are involved in the conformational transition of PrPC to PrPSc. ${ }^{22-27}$ The studies by Chen W. et al. ${ }^{9}$ and Chen J. et al. ${ }^{28}$ have stated that the residues 173-226 comprising of mainly the helices $\mathrm{H} 2-\mathrm{H} 3$, play a crucial role in the conformational transitions. The studies by Adrover et al. ${ }^{29}$ Chakroun et al., ${ }^{30}$ Tycko et al. ${ }^{31}$ and Dima et $a l .{ }^{32}$ have suggested the role of $\mathrm{H} 1$ and $\mathrm{H} 3$ in the conversion of PrPC to PrPSc. Role of tertiary/long range interactions between St2-H2 loop and H3 have been found to be crucial to understand the early events in the misfolding process. ${ }^{33,34}$ The stability of PrPC is mostly attributed to its hydrophobic core and hydrogen bonds. ${ }^{35-38}$ Hence pathogenic mutations residing in the hydrophobic core of PrPC display different effects on the mature protein. ${ }^{35}$ Besides amino acid point mutations, the $\mathrm{pH}$, and temperature also affects the thermodynamic stability of the protein and lead to pathological conformational transformations. ${ }^{39-42}$ The understanding of how various mutations affect the conformational changes in prion protein would unveil the mechanism by which familial mutations facilitate the pathogenic process.

Hence, in this work molecular dynamics of wild type, a pathogenic mutant $\mathrm{E} 200 \mathrm{~K}$ responsible for the most common familial form of Creutzfeldt-Jakob disease ${ }^{16,43,44}$ and a non-pathogenic mutant E219K known to provide extra stability to PrP have been studied. ${ }^{45,46}$ Although the point mutations (glutamate to lysine) are identical but occur at different positions (one at position 200 and another at position 219) and show contrasting effects i.e. one being pathogenic (E200K) and another providing extra stability (E219K). In order to understand the structural changes responsible for difference in their functional behaviour due to these mutations at different location, molecular dynamics simulations have been performed. For each of these variants four sets of simulations of $200 \mathrm{~ns}$ have been carried out. We have tried to address the structural changes which may be responsible for pathogenicity. Also we have tried to determine which of structural changes may be responsible to provide extra stability to protein. The main aim of the study is to understand early events in the wild type, E200K and E219K prion variants which may triggers PrPC to PrPSc conversion. Various structural parameters have been calculated for these simulations data and using these parameters, feature based correlation analysis has been done. One of the feature from the correlation analysis has been used for Markov State Model (MSM) calculation, which has helped to study the intermediate states and the kinetics of transitions involved between them. MSM have been previously used to understand folding/unfolding and $\mathrm{f}$ behaviour of protein. ${ }^{47-50}$

\section{Method molecular simulations}

\subsection{Simulation protocol}

The NMR structure PDB id 1QM0 with the residue range 125228 was selected as the starting structure for the simulations as the residues 23-124 are known to be highly disordered and unstructured. The two variants of the prion protein were obtained by replacing glutamate with lysine at position 200 and another at position 219 respectively using Chimera. ${ }^{51}$ Molecular dynamics simulations were carried out using GROMACS v5 (ref. 52) with AMBER ff99sb force field ${ }^{53}$ for $200 \mathrm{~ns}$ (four sets) for all the three systems. All the systems were solvated in a cubic box with TIP3P ${ }^{54}$ water. The distance between closest protein fragment and edges of the box was kept to be $9 \AA$. All the systems were neutralized by adding $\mathrm{Na}^{+}$ions. For wild type three $\mathrm{Na}^{+}$ ions were added for $\mathrm{E} 200 \mathrm{~K}$ and $\mathrm{E} 219 \mathrm{~K}$ one $\mathrm{Na}^{+}$were added. The energy minimization was carried out for 2500 steps. This was followed by two step equilibration, the first one was position restrained NVT equilibration for 500 ps using V-rescale thermostat. ${ }^{55}$ This was followed by NPT equilibration for $1 \mathrm{~ns}$ using the Berendsen barostat. ${ }^{56}$ Long-range electrostatic interactions were calculated using the particle-mesh Ewald (PME) method. ${ }^{57}$ A $12 \AA$ cut-off was set for coulombic and van der Waals's interactions. Newton's equation of motions was solved using the Leap frog integrator with the integration time step of 2 fs. The LINCS algorithm ${ }^{58}$ was used to constrain the bond lengths involving in hydrogen atoms. The production runs for $200 \mathrm{~ns}$ were carried out using Parrinello-Rahman barostat. ${ }^{59}$

\subsection{Analysis}

GROMACS analysis utilities, were used for the analysis. The hydrogen bonds were calculated using g_hbond utility of GROMACS where interactions with distance $<0.35 \mathrm{~nm}$ between cooperated donor and acceptor heavy atoms were considered. GROMACS trajectory was converted to PDB trajectory and native contacts analysis was done using cpptraj utility of AMBER Tools using contact criteria of $0.35 \mathrm{~nm} .{ }^{60} \mathrm{VMD}$ was used for visualization and image rendering. ${ }^{61}$

\subsection{Markov state models analysis (MSM)}

Markov state models (MSM) is an important tool for predicting interesting intermediate states and kinetics between them from molecular dynamics data of a biological system. Creation of states and the transition matrix depends on the choice of collective variable (CVs) and the lag time. ${ }^{62-65}$ MSM calculations were done using Pyemma software package. ${ }^{66}$ Following steps were carried out to build the MSM; (a) VAMP score calculation was done for different features, which is based on variational principle. ${ }^{67}$ The variational principle helps to check the robustness of MSM. VAMP score were calculated for following features viz. dihedral angle, positions (coordinates) and residue min_dist (mindist). (b) Feature selection (CV), wherein the residue minimum distance (mindist) was used as the featurizer. (c) Computing of time independent component analysis (TICA) using this featurizer as the second step. TICA helps to reduce dimensionality of the trajectory data. (d) Clustering of MD data 
using $\mathrm{K}$ means clustering and (e) creation of macrostate (states) using PCCA. (f) Transition matrix was built using chosen lag time in such a way that the transition matrix follows Markovian behaviour. Eigen decomposition of transition matrix results in eigenvectors which represents slow motions in the systems. The fluxes and mean passage time calculations are done from computing transition pathways between states by discrete transition path theory. ${ }^{68}$ The choice of lag time is related to eigenvalue which, gives us physically measurable timescale know as implied timescale $\left(t_{\mathrm{i}}\right)$ using eqn (1).

$$
t_{\mathrm{i}}=-\frac{\tau}{\ln \lambda_{\mathrm{i}}}
$$

where $t_{\mathrm{i}}$ is implied timescale, $\tau$ is the lag time and $\lambda_{\mathrm{i}}$ is an eigenvalue. The validation of the MSM was done using Chapman-Kolmogorov equation ${ }^{62}$ given by the eqn (2)

$$
T(n \tau)=T(\tau)^{n}
$$

where $n$ is an integer number of steps, $T(\tau)$ is transition matrix at lag time $\tau$.

The validated MSM model is very useful to understand intermediate states and kinetics between them. All these steps of MSM were carried out for all the three variants.

\subsection{Electrostatic potential calculation}

The electrostatic interactions within the protein plays important role in maintaining the stable structure of protein. Any change in the surface charge may alter the protein interaction with solvent and other biomolecules in the near environment. This can affect stability of protein. In the current study, ESP for all the three variants were calculated using Poisson-Boltzmann equation. The electrostatic potential $\psi(r)$ for a simple system of charged particles in solvent was calculated with spacedependent dielectric constant $\varepsilon(r)$ and the charge density $\rho(r)$ of the solute. Here for ESP calculation, solvent was considered as continuous medium with an exterior dielectric constant $\left(\varepsilon_{\text {ext }}\right.$ $=80$ ) and the prion protein was treated as charged particles in the solvent with an interior dielectric constant $\left(\varepsilon_{\text {int }}=4\right)$. The electrostatic potentials were calculated using programs PDB2PQR, ${ }^{69}$ APBS $^{70}$ and PROPKA. ${ }^{71}$ APBS calculations were done form the MSM states. Visualization of APBS calculations were done using VMD.

\section{Results and discussions}

Four sets of simulations have been carried out for $200 \mathrm{~ns}$ each for wild type prion, pathogenic mutant (glutamate to lysine) at position 200 and non-pathogenic mutant (glutamate to lysine) at position 219. Henceforth, wild type system has been represented as WT and mutant at position 200 and 219 have been represented as E200K and E219K respectively. Feature based correlation analysis is done for first trajectory to identify reaction coordinate for the MSM. MSM analysis is carried out using all the four sets of simulation for WT, E200K and E219K the prion variants. Starting structure for all the three variants has been shown in the Fig. 1 with the mutated residue represented as ball and stick. Most of the knowledge about prion protein is available in the form of NMR structures. Hence, the backbone chemical shifts were calculated for simulated systems using 'Shift2x' software. ${ }^{72}$ The chemical shifts were calculated for all the WT and mutants. The results of the simulations were compared with that of experimental values of chemical shifts and are presented in the Fig. 2. It is clear that the experimental shift values are in well agreement with that of all the three simulated prion variants. These suggests that the systems have been well equilibrated.

\subsection{Correlated factors in prion dynamics}

To identify which of the structural parameters are correlated and important in discrimination of prion variants, an $\mathrm{R}$ based utility has been used. ${ }^{73}$ It uses spectral based decompositions to examine the covariances/correlations between parameters/ features. The features used to carry out this analysis were RMSD, RGY, SASA, native contacts, residue distances, mainchain-mainchain (MM) hydrogen bonds, mainchain-sidechain (MS) hydrogen bonds and sidechain-sidechain (SS) hydrogen bonds. The details of structural parameters have been given in the ESI (Fig. S1-S7†). Before doing spectral decomposition, clustering correlation was done using the above mentioned parameters (Fig. 3A). The clustering correlation gives an idea about how the different parameter are correlated with each other. A positive value indicates positive correlation and a negative value indicates negative correlation. The maximum value for positive and negative correlation can be 1 and -1 respectively. From the Fig. 3A, maximum positive correlation is seen between RGY and SASA.

The next step after clustering correlation is the feature based PCA to identify the dominant features contributing in the protein dynamics. The Fig. 3B shows the results for feature based PCA for all the three variants. In feature based PCA, clear separation within the population of conformers was seen between different prion variants (Fig. 3B). Wild type (blue colour) and E219K (green colour) were clustered together whereas E200K (orange colour) formed a separate cluster. The arrows shown in the Fig. 3B suggests that native contacts and hydrogen bonds (MM) were positively correlated while native contacts and distance features were negatively correlated. From the above clustering, it was clear that E219K had correlated structural properties as observed for WT. However, E200K behaved differently as compared to WT. The features like distance, native contacts and mainchain-mainchain hydrogen bonds played dominant role to discriminate E200K and WT, E219K systems. This analysis has helped to find the collective variable for building the MSM. The distance (minimum distance) feature has been used as a collective variable to find states in the MSM analysis.

\subsection{Prion dynamics: interesting intermediate states and kinetics}

MSM analysis helps to understand kinetics followed by a system from several discrete simulations data. ${ }^{74}$ The selection of lag time plays an important role in construction of a Markov model. 

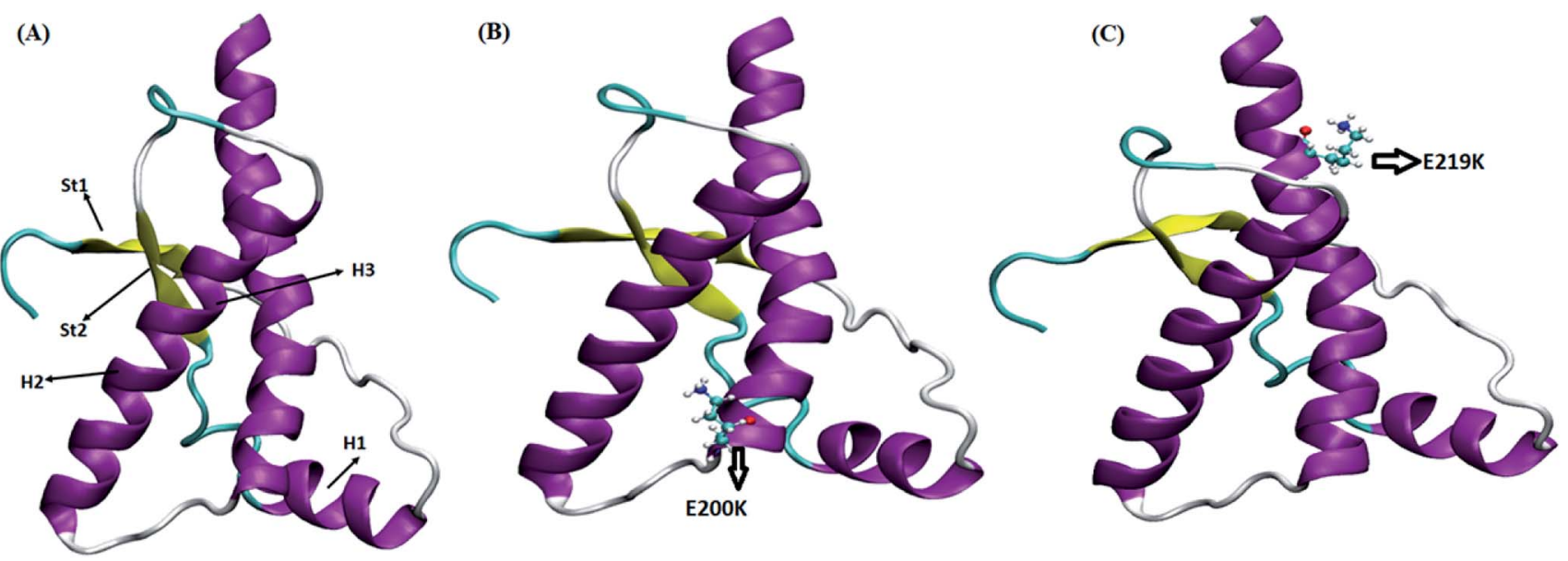

Fig. 1 Starting structure used for simulation with mutant residue represented as ball and stick. (A) Wild type. (B) E200K. (C) E219K.

The lag time for building the macro state was calculated by plotting implied timescale (ESI Fig. S8a†). The lag time of $20 \mathrm{~ns}$ was chosen for all the three variants since, it attains saturation after 20 ns. VAMP score was calculated for different features. It helps to check the robustness of the MSM. VAMP score were calculated for following features viz. dihedral angle, positions
(A)

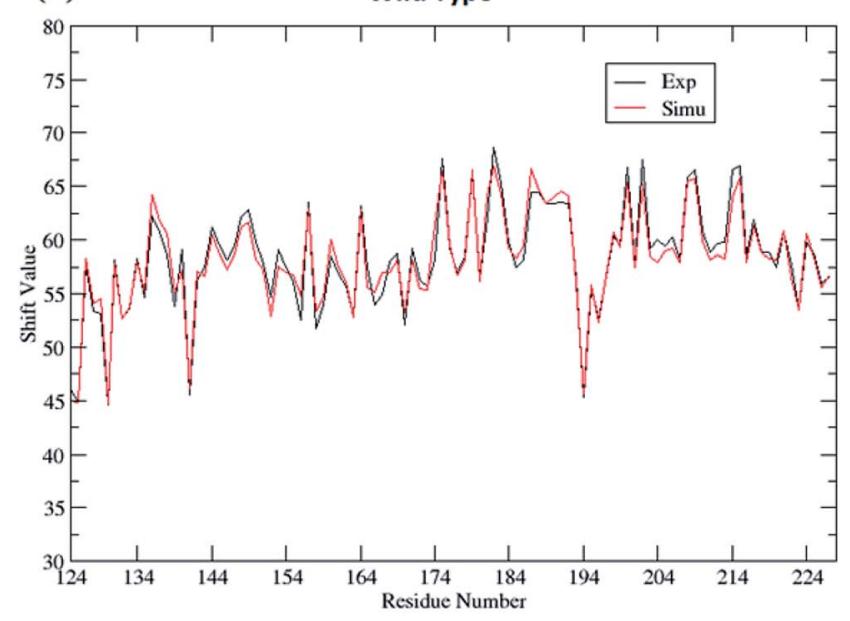

(B)

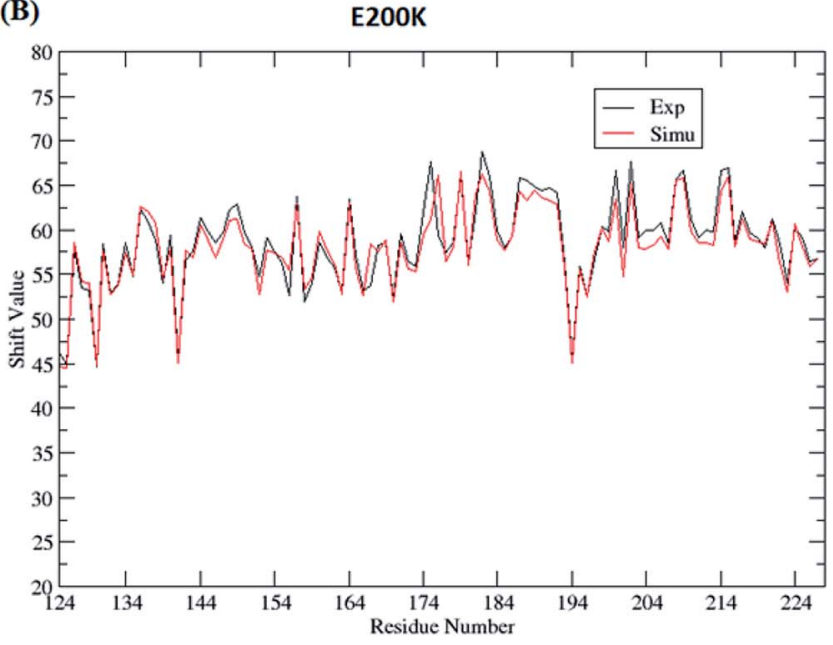

(C)

E219K

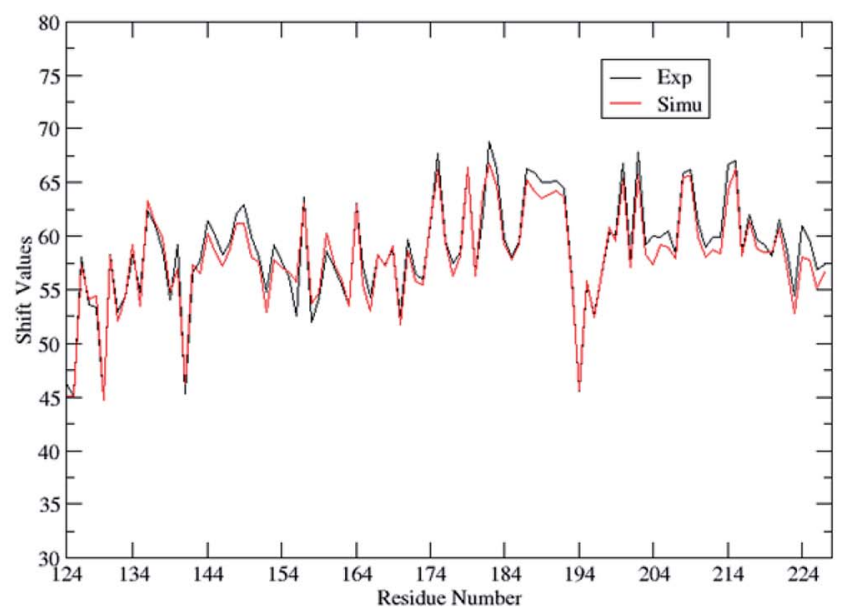

Fig. 2 Comparison of chemical shift values between experimental and simulated ensemble structure for all the three variants. 

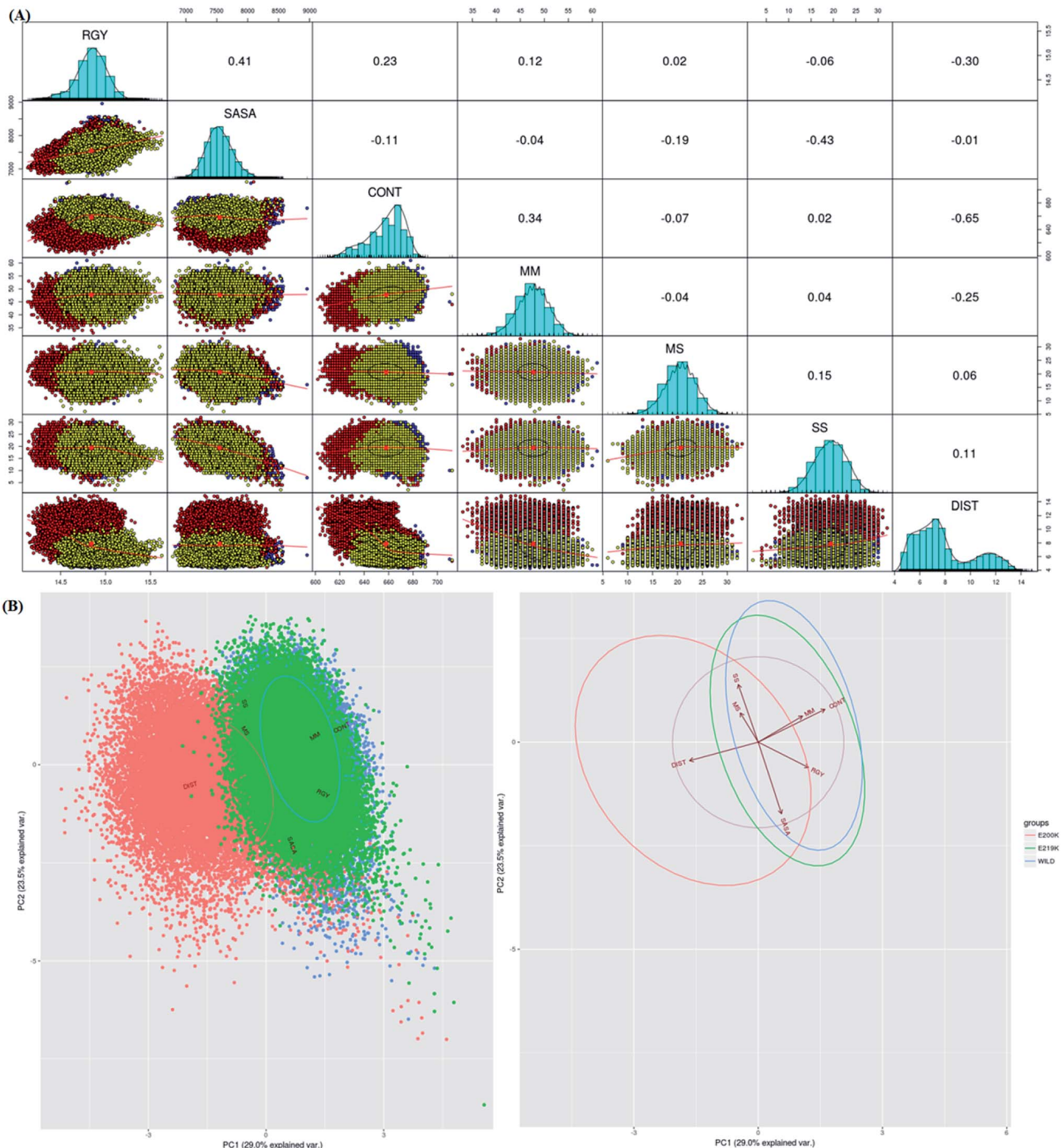

Fig. 3 (A) Correlation plot between various parameters. Upper triangle represents the correlation value between different parameters. Lower triangle shows clusters where blue colour is wild type, red colour E200K variant and yellow colour is for E219K variant. (B) PCA cluster using defined list of parameters (RMSD, RGY, SASA, native contacts, hydrogen bonds, and important contacts). Blue colour is for wild type, orange colour is for E200K variant and green colour is for E219K variant.

(coordinates) and residue min_dist ESI Fig. S8b.† VAMP score was maximum for residue min_dist CV. Validation of Markovian behaviour of MSM model is tested through the Chapman-Kolmogorov ${ }^{62}$ equation. This gives an idea about the probability of decay observed for the major metastable states predicted by MSM. The ESI Fig. S9† has been given to show the validation of MSM model using the Chapman-Kolmogorov equation. In order to get the kinetics of the system, the trajectories from all the four runs were used to build MSM. In case of the WT, the spread of conformation along first time independent component (tIC) was from -0.5 to 2 and -2 to 1 along second tIC component has been seen in the Fig. 4. TICA 

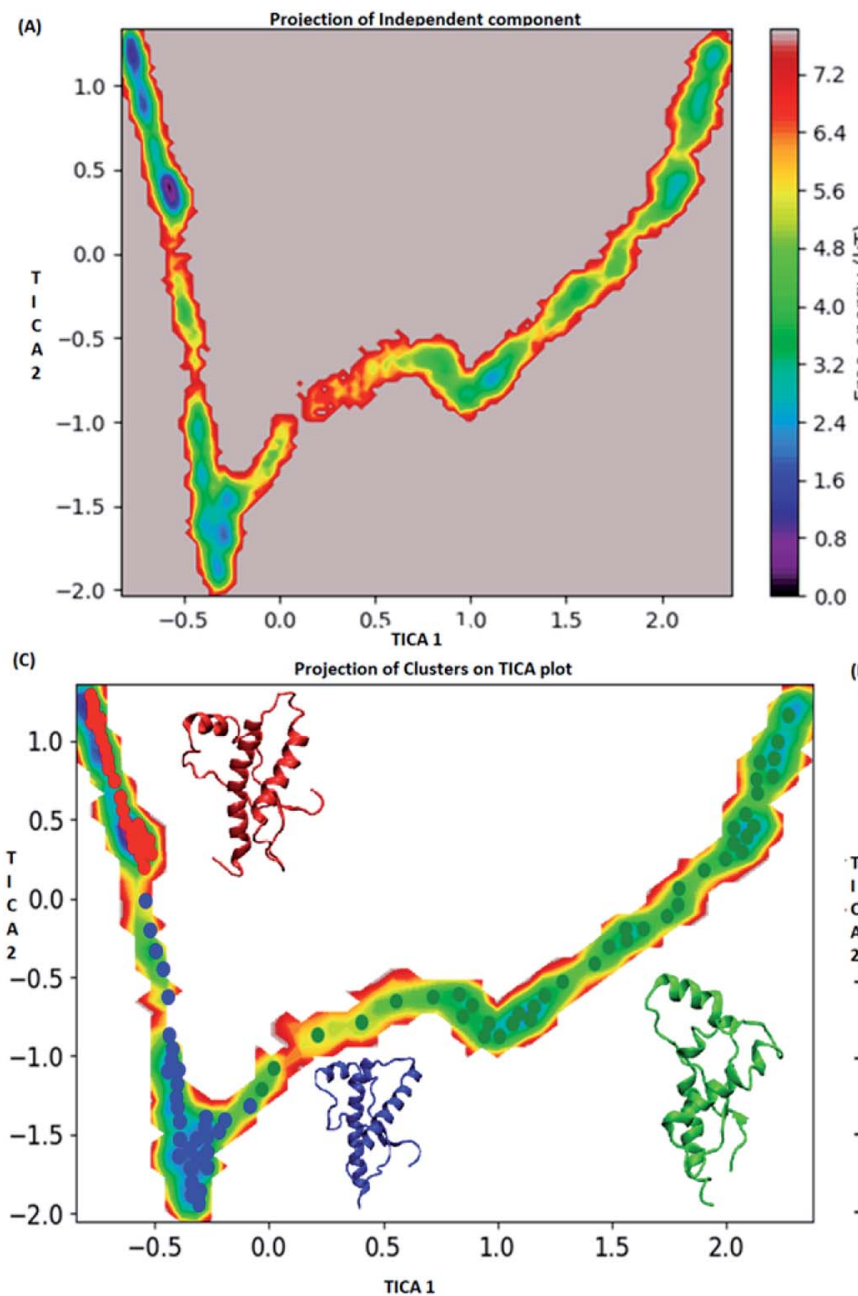

Fig. 4 Markov state model analysis for wild type. (A) Free energy landscape along first two time independent component. (B) Projection of independent component. (C) Free energy landscape along first two time independent component showing three major states (red, blue and green) along with their representative structure. (D) Free energy landscape along first two time independent component showing pathway followed to reach unstable state (green colour) from stable state (red colour).

clustering followed by Perron-cluster cluster analysis (PCCA) macrostate clustering resulted into three metastable states which have been shown in the Fig. 4C. The state 1 (red colour) encompasses of the structure that belongs to the low energy basins. The state 2 represented in blue colour encompasses the structure within an energy range of 2.4 to $4.8 \mathrm{kT}$. The state 2 was separated from state 1 by an energy barrier of 5.6 to $6.4 \mathrm{kT}$. The third state (green colour) consists of conformation with slightly higher energy ( 4.8 to $6.4 \mathrm{kT}$ ). The structures in state 1 had wellformed secondary structure components (ESI Fig. S10†). Similar to state 1, state 2 also had conformers with well-formed secondary structures. However in the state 3 the conformers showed deformed secondary structures. There was loss in helicity of $\mathrm{H} 1$ along C-terminal region; $\mathrm{H} 3$ also showed deformation along C-terminal region. Markov state model analysis also provides an insight on transitions between states. It gives the probability of the protein conformations traversing from one state to another. It was observed that the structure tends to go from state 1 to state 2 and finally reaching state 3 . The mean
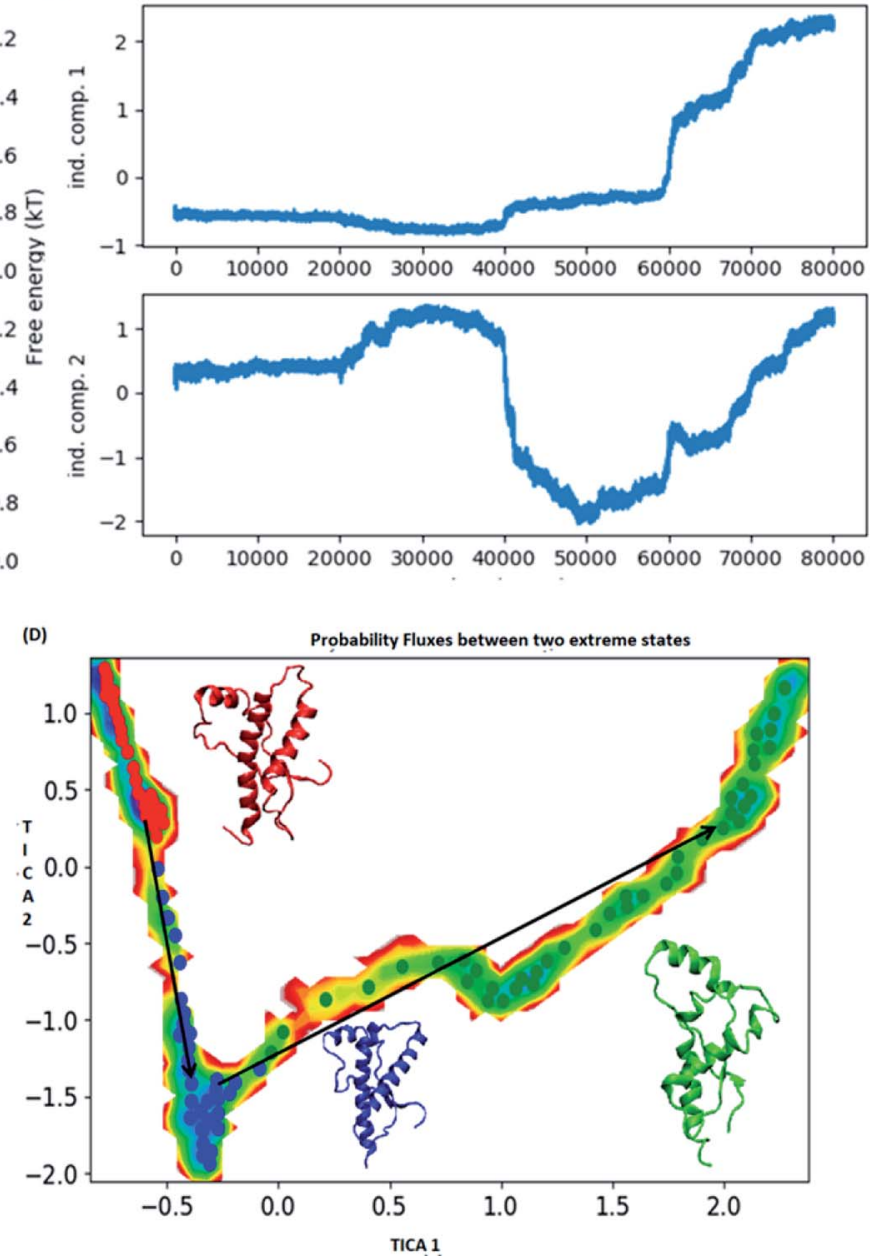
(A) Proiection of Independent component

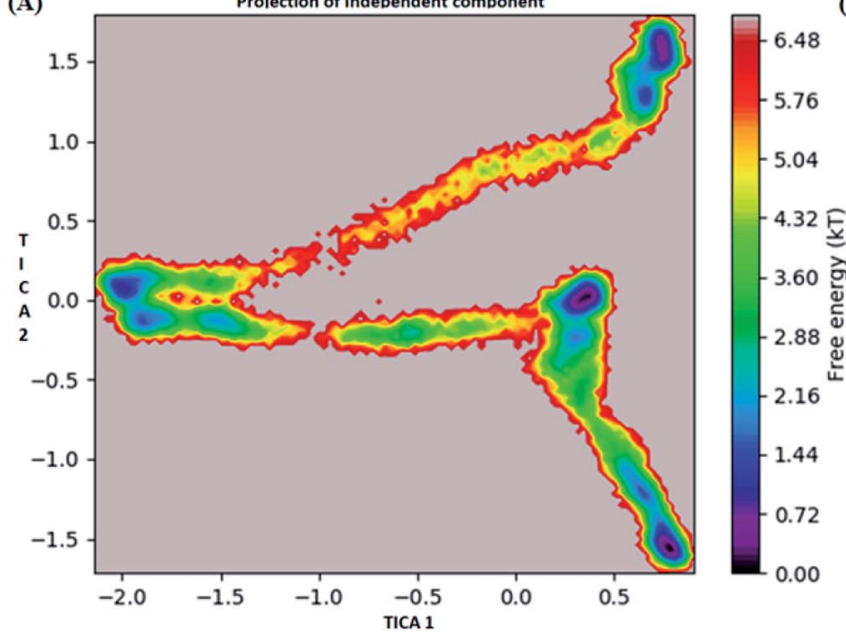

(C)

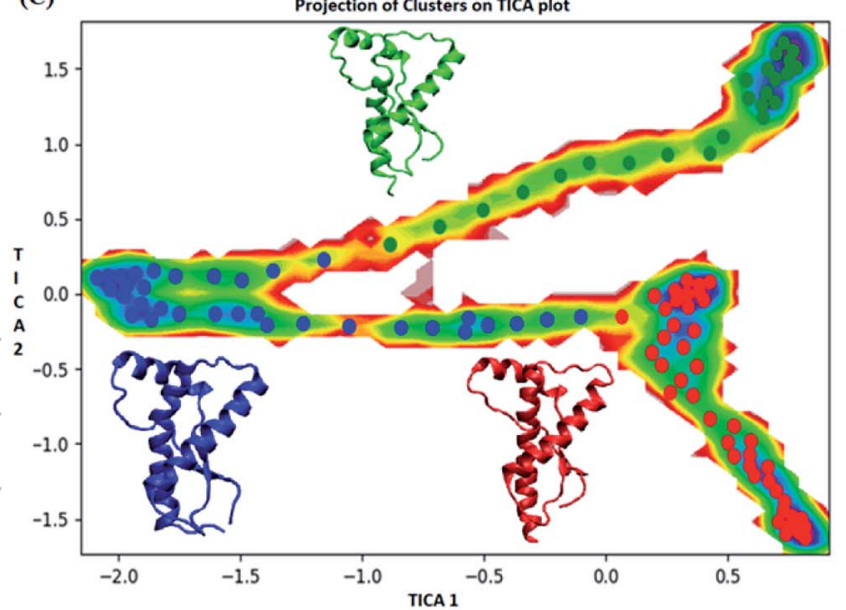

(B)
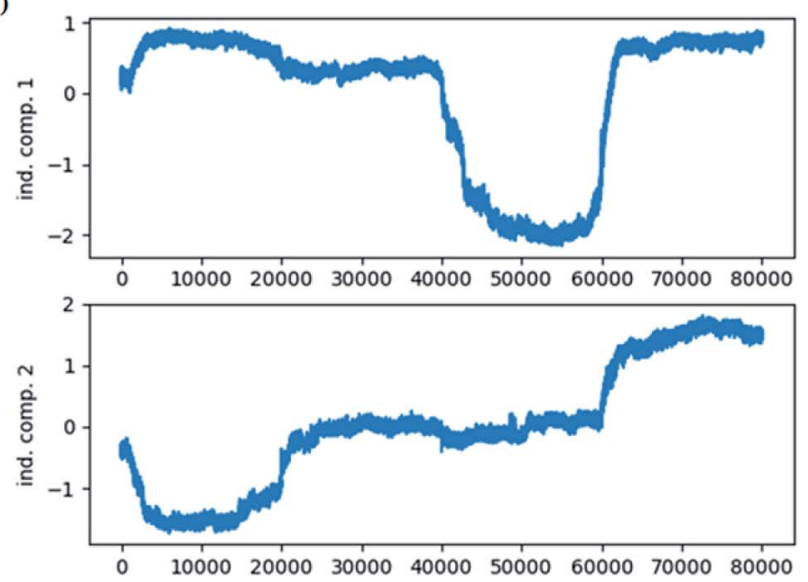

(D)

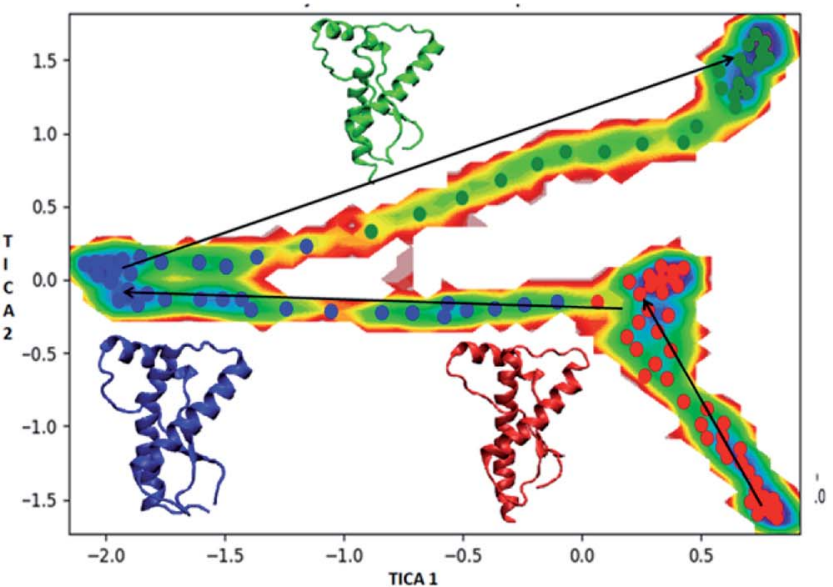

Fig. 5 Markov state model analysis for E200K variant. (A) Free energy landscape along first two time independent component. (B) Projection of independent component. (C) Free energy landscape along first two time independent component showing three major states (red, blue and green) along with their representative structure. (D) Free energy landscape along first two time independent component showing pathway followed to reach unstable state (green colour) from stable state (red colour).

had intermediate structures and state 3 had more deformed conformations.

The pathway suggest that native like structures in state 1 tends to undergo slight deformation and reach the state 2 . This deformation starts with loss of $\mathrm{H} 2$ followed by deformation of $\mathrm{H} 3$ at both $\mathrm{C}$ and $\mathrm{N}$ terminal end as observed in state 3 . The mean passage time for the system from stable structure to deformed structure was $1.5 \mathrm{~ms}$ whereas for the reversible process it was $380 \mathrm{~ms}$. For the E219K, the spread of conformation along first and second tIC has been shown in the Fig. 6 . Three major states were seen, state 1 (red colour) which laid at tIC1 tIC2 $(-0.5,0.7)$ and had energy value of $\sim 0.72 \mathrm{kT}$. The state 2 (blue colour) consisted of population with energy varying in range of 0.72 to $2.8 \mathrm{kT}$. The state 1 and state 2 were separated by energy barrier of $\sim 3 \mathrm{kT}$. The structures in the state 3 (green colour) had energy in the range of 1.6 to $6 \mathrm{kT}$. The structures in state 1 had well-formed secondary structures (ESI Fig. S12†). The structures in state 2 were opened up. The state 3 had deformed $\mathrm{H} 1$ and $\mathrm{H} 3$ and the structures were more compact.
Thus, the state 1 had native like conformation, state 2 had intermediate conformations and state 3 had deformed conformations.

The mean passage time for going from stable structure to deformed structure was predicted to be $750 \mathrm{~ms}$, whereas the same for reversible time is $650 \mathrm{~ns}$. From MSM study it was clear that the E200K mutant was easily destabilized and had a very low mean passage time. The mean passage time was observed to be the longest for E219K mutant among the three variants. Hence, it is clear from MSM mean passage time that the E200K variant can be deformed at a faster rate as compared to be wild type and E219K variant. Few geometrical parameters like native contacts and secondary structure elements were analysed for MSM states of all three systems and shown in the ESI Fig. S10S13. $\uparrow$ The native contacts for WT and E219K systems were found to be in the range of 640 to 660 (ESI Fig. S13A and S13C $\dagger$ ) respectively. But the number of native contacts for E200K system were found to be in the range of 620 to 660 (ESI Fig. S13B $\dagger$ ). The number of native contacts were the highest for 

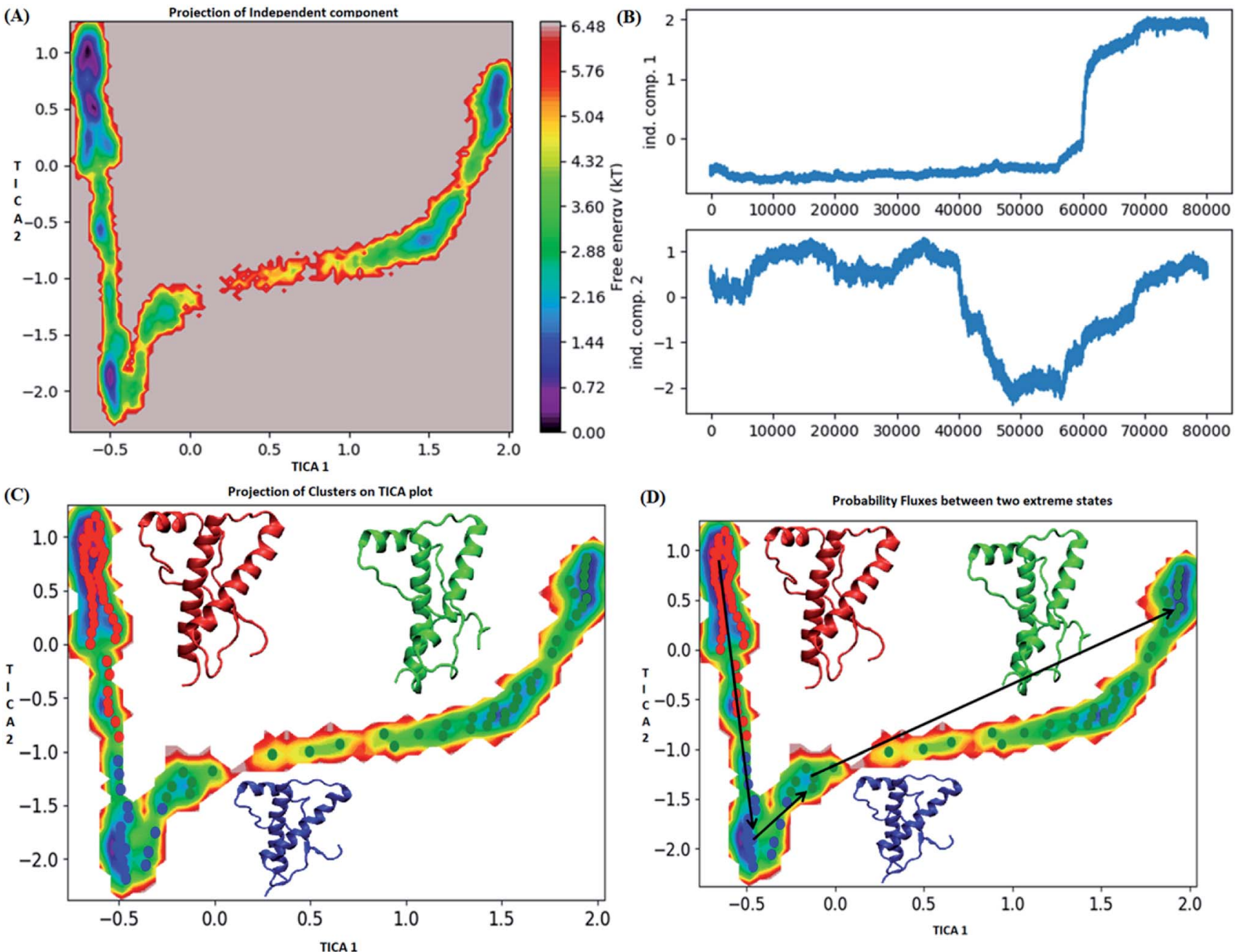

Fig. 6 Markov state model analysis for E219K variant (A) free energy landscape along first two time independent component. (B) Projection of independent component. (C) Free energy landscape along first two time independent component showing three major states (red, blue and green) along with their representative structure. (D) Free energy landscape along first two time independent component showing pathway followed to reach unstable state (green colour) from stable state (red colour).

state 1 and lowest for state 3 in all the three systems. MSM was able to predict that the intermediate states and the conformations obtained in state 3 may be prone towards protein aggregation. The secondary structure analysis for the structures of MSM states of all the systems have been shown in the ESI Fig. S10-S12. $\dagger$ The state wise secondary structure analysis has suggested that state 3 structure showed highest loss in secondary structures as compared to the states $1 \& 3$ in all the three systems. Thus in cluster 3 WT and E219K variant showed loss in secondary structure of $\mathrm{H} 1$ and $\mathrm{H} 3$ while for E200K loss in secondary structure was observed in $\mathrm{H} 2$ and $\mathrm{H} 3$. Thus it may be inferred that the early deformation of $\mathrm{H} 2$ may be crucial for faster conversion of PrPc to PrPSc.

\subsection{Important contacts and fluctuations}

In-order to find out which of residues were affected during unfolding process of prion protein, residue-wise native contacts were plotted for each of the clusters/states of MSM. Fig. 7 shows the residue-wise native contacts for all the three clusters/states in the all three variants. For cluster 1 , the residue-wise native contacts did not vary much for all the three variants (Fig. 7A). The native contacts for the residue numbers 146, 163, 193, 194, 200 and 204 of E200K prion variant have been affected as compared to that of WT and E219K. For the cluster 2, major changes have been seen in the loop region connecting $\mathrm{H} 2$ and H3. H3 region also showed decrease in number of residue-wise native contacts in E200K as compared to WT and E219K as seen in the Fig. 7B. In the cluster 3, the difference in number of residue-wise native contacts was more prominent between all three systems. The E200K variant showed further decrease in the number of native contacts for $\mathrm{H} 2$, the loop connecting $\mathrm{H} 2$ and $\mathrm{H} 3$, and $\mathrm{H} 3$ (Fig. 7C).

Further, important salt bridges/contacts in the H2 \& H3 regions were measured in all the three clusters/states of WT, E200K and E219K systems. The distances of important salt bridges have been plotted in the ESI Fig. S14 to S17. $\uparrow$ The ESI Fig. S14-S17† shows distance plot between residues 146-208, 

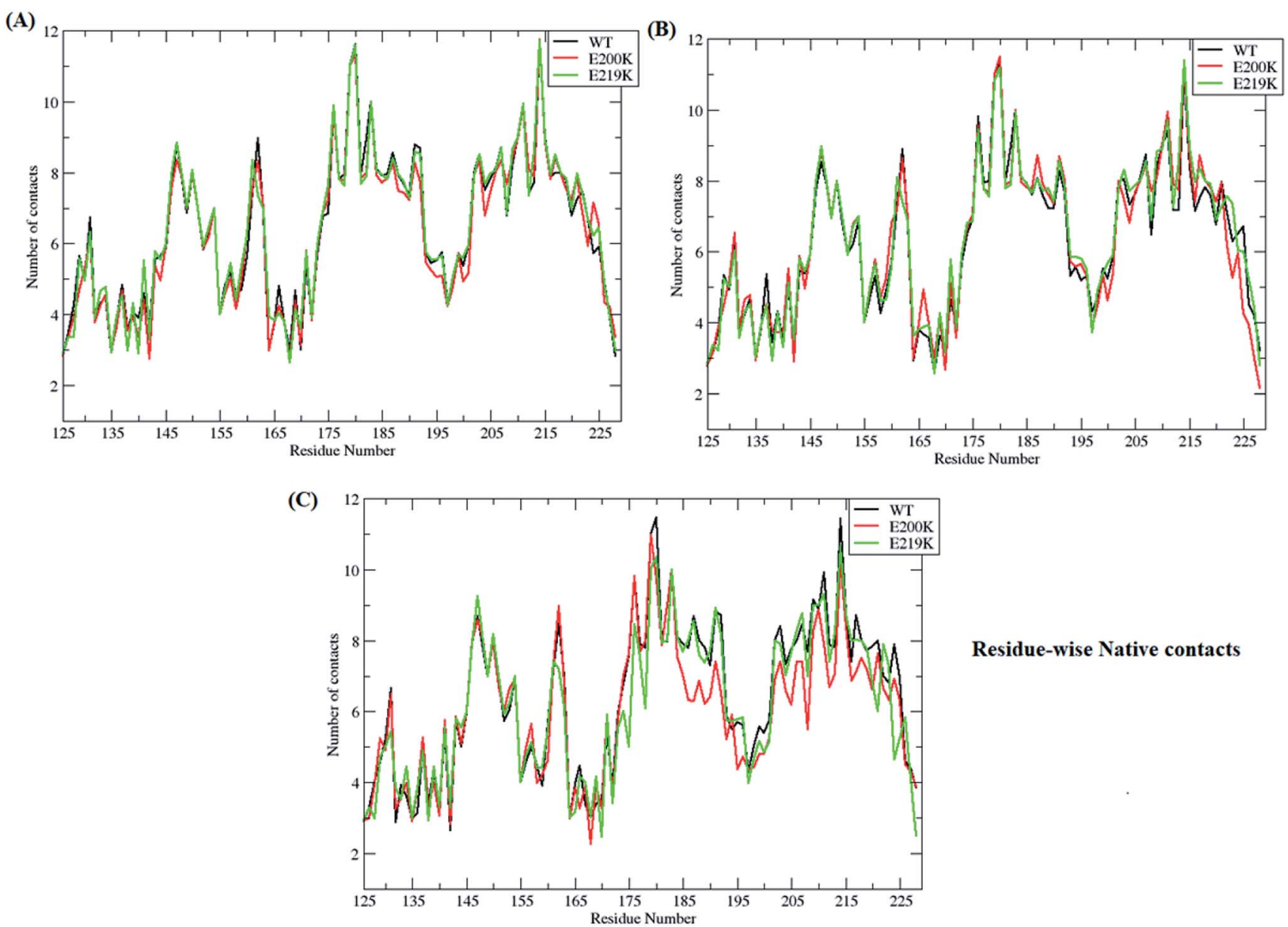

Residue-wise Native contacts

Fig. 7 Figure showing residue-wise native contacts for MSM states (A) residue-wise native contacts for state 1 for all the three variants (B) residue-wise native contacts for state 2 for all the three variants $(C)$ residue-wise native contacts for state 3 for all the three protein variants.

149-202, 156-196 and 200-204 residues respectively. For contact between residues 146 and 208, the distance was maintained around 9-10 $\AA$ in all the three MSM states of E200K, while in case of WT and E219K the distance between these residues gradually increased while traversing from MSM state 1 to 3. In WT and E219K systems, the distance was observed around 4-6 ̊ for cluster 1, around 7-8 A for cluster 2 , while it was around $10 \AA$ in the cluster 3 . Similar trend was observed for distance between residue 149 and 202. For E200K, the distance was maintained around $9-10 \AA$ in all three MSM clusters. For WT, it showed gradual increase from $\sim 4 \AA$ (cluster 1 ) to $\sim 10 \AA$ (cluster 3). Similar trend was observed for E219K system. The contact between residues 156 and 196 and 200 and 204 have similar behaviour like the earlier contacts for all the E200K, WT and E219K systems. In E200K the disruption of contacts was observed in all the three states/clusters while in WT and E219K it showed gradual disruption while traversing from state 1 to state 3. Thus, the E200K mutation may lead to early disruption of some of the salt bridges which may be one of the reasons for the pathogenicity of E200K. Also these salt bridges were stronger in E219K (smaller distance between the residues) which may be the reason for extra stability.

\subsection{Structural response to mutations}

Borgohain et al. ${ }^{75}$ discussed the conformational changes occurring due to the two pathogenic mutants T193I and R148H using classical molecular simulations. They did not observe any major structural transition, except for few local variations. For T193I mutant they observed depletion of $\beta$-sheet content and conversion of a $3^{10}$ region into a coil, whereas for $\mathrm{R} 148 \mathrm{H}$ observed $\beta$-sheet depletion and appearance of new $3^{10}$ region in $\mathrm{H} 2$. They also observed conversion of two $\alpha$ helical regions into two new turn regions in $\mathrm{H} 2$ and $\mathrm{H} 3$ helices. Chebaro et al. ${ }^{76}$ studied effect of T183A mutation both on monomer and dimer through coarse grained molecular simulations. They observed that $\mathrm{H} 1$ was robust and the $\mathrm{H} 2 / \mathrm{H} 3$ subdomain showed higher propensity for intra- and inter- $\beta$-sheets. Zhou et al. ${ }^{77}$ carried out molecular dynamics simulation studies to study effect of G127V substitution on dimer formation. They observed that the V127 variant reduces main-chain $\mathrm{H}$-bond interaction in St1 region, making it unfeasible for making $\beta$ strand structure and thereby prevent dimer formation. DeMarco et al. ${ }^{78}$ studied the effect of low $\mathrm{pH}$ on prion protein through molecular dynamics simulations. They observed that at normal pH the structure remains stable but at low $\mathrm{pH}$ critical long-range salt bridges were broken 

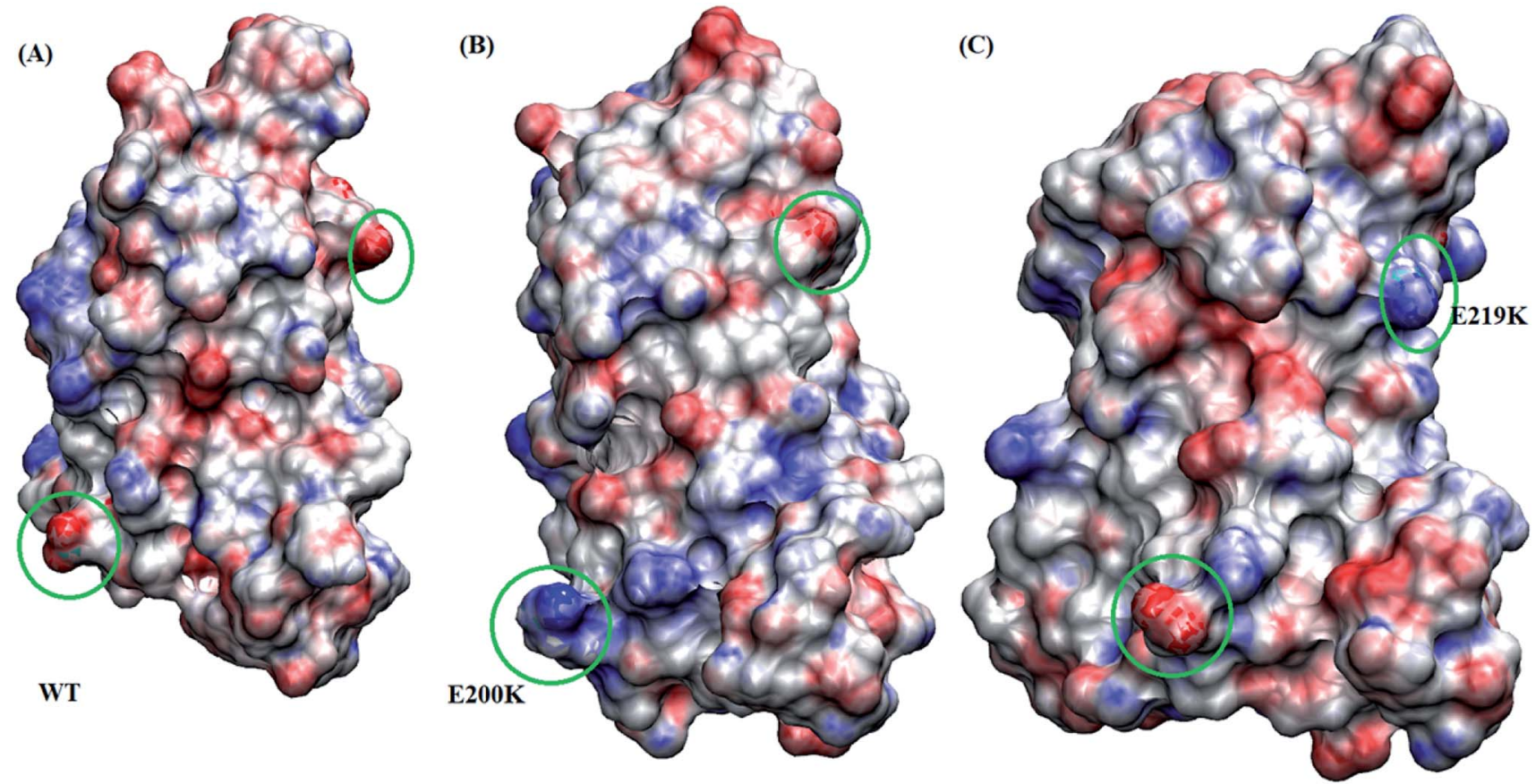

Fig. 8 Figure showing electrostatic potential for all the three protein variant. Red is negatively charged surface and blue being positively charged surface. (A) Wild type. (B) E200K. (C) E219K.

resulting in destabilized structures. They also concluded that the breakage of salt bridges led to disconnection of $\mathrm{H} 1$ from $\mathrm{H} 2-\mathrm{H} 3$ domain. The results presented here also suggest the weakening of salt bridges and local variation in the $\mathrm{H} 2-\mathrm{H} 3$ domain, may act as early events in the prion aggregation.

Studies carried out by Biljan et al. ${ }^{45}$ on E219K and Zhang et al. ${ }^{16}$ on E200K mainly suggested the role of surface charge as important factor for extra stability of E219K and pathogenicity of E200K mutants. In the current study, electrostatic potential (ESP) for all the three variants are calculated using PoissonBoltzmann equation. The Fig. 8 shows ESP snapshot of MSM state 3 structures of WT, E200K and E219K. The charge on the surface of protein has been represented by positively charged (blue colour) and negatively charged (red colour). For E200K, the surface tends to become positively charged at the site of mutation. For E219K, the surface showed subtle alteration in the charge at the site of mutation. These differences in the charges of the mutant systems may be playing a role in the disruption of the native contacts. The two mutations tend to perturb the surface charge distribution and this change may affect the aggregation propensity of protein as discussed in the literature. ${ }^{44,45}$ In the simulation reported here we observed perturbation in the surface charge as reported for E219K and E200K. As charge plays an important role in maintaining electrostatic interactions, the change in surface charge may also affects the salt-bridges interactions, which may lead to destabilization in E200K and stabilization in E219K. As reported by previous simulation of DeMarco et al.,$^{78}$ Menon et al. ${ }^{79}$ the breakage of salt bridges leads to a cascade of events which further causes the destabilization of other intra helical contacts. Rossetti $e t a l{ }^{80,81}$ carried out simulation study for WT, E200K,
E219K and Q212P and reported difference in salt bridge interactions among various prion variants in there study. In our simulation we observed the difference among the following salt bridges viz. ARG156-GLU196, GLU146-ASP202, ARG156ASP202, GLU/LYS200-LYS204. We also observed similar behaviour wherein breakage of important contacts may have decreased in the native contacts in E200K and thereby increased random motion between the helices. All these structural variations and destabilization effects have been dominant in E200K as compared to E219K and WT.

\section{Conclusion}

The aim of the study was to detect the early structural changes in all the three variants which were responsible for the stability and conversion from PrPC to PrPSc. The MSM based analyses were carried out to find out the differences between E200K, E219K and WT systems. The MSM analysis were able to predict the intermediate states which helped to understand the effect of same amino acid mutation at two different locations. The structural and kinetics information from MSM states were able to predicted faster destabilization of E200K variant as compared to WT and E219K. Native contacts and secondary structures were found to be affected in E200K as compared to E219K and WT systems. The perturbation in surface charge distribution may have influence the tertiary interactions between secondary structures in the prion. In E200K, the residues lying in $\mathrm{H} 2$ and $\mathrm{H} 3$ regions tend to show reduction in the number of native contacts. The breakage of these important contacts in E200K may be the reason for increased fluctuation of $\mathrm{H} 2$ and $\mathrm{H} 3$ helices. These proposed MSM intermediate states in all the 
three prion systems may prove to be useful in structural differentiation of as early events occurring in the conversion of PrPC to PrPSc form.

\section{Conflicts of interest}

There are no conflicts to declare.

\section{Acknowledgements}

The authors gratefully acknowledge the Ministry of Electronics and Information Technology (MeitY), Government of India, New Delhi, and National Supercomputing Mission (NSM) for providing financial support. This work was performed using the "Bioinformatics Resources and Applications Facility (BRAF)". I would like to also thank Ms Shruti Koulgi for improving the manuscript.

\section{References}

1 S. Liemann and R. Glockshuber, Transmissible spongiform encephalopathies, Biochem. Biophys. Res. Commun., 1998, 250(2), 187-193.

2 S. B. Prusiner, Scrapie prions, Annu. Rev. Microbiol., 1989, 43(1), 345-374.

3 R. Linden, V. R. Martins, M. A. Prado, M. Cammarota, I. Izquierdo and R. R. Brentani, Physiology of the prion protein, Physiol. Rev., 2008, 88(2), 673-728.

4 A. Aguzzi and A. M. Calella, Prions: protein aggregation and infectious diseases, Physiol. Rev., 2009, 89(4), 1105-1152.

5 J. R. Greig, Scrapie in sheep, J. Comp. Pathol. Ther., 1950, 60, 263-266.

6 S. B. Prusiner, Molecular biology of prion diseases, Science, 1991, 252(5012), 1515-1522.

7 C. Weissmann, Molecular genetics of transmissible spongiform encephalopathies, J. Biol. Chem., 1999, 274(1), 3-6.

8 S. B. Prusiner, Prions, Proc. Natl. Acad. Sci. U. S. A., 1998, 95(23), 13363-13383.

9 W. Chen, M. W. Van Der Kamp and V. Daggett, Diverse effects on the native $\beta$-sheet of the human prion protein due to disease-associated mutations, Biochemistry, 2010, 49(45), 9874-9881.

$10 \mathrm{Y}$. Wen, et al., Unique structural characteristics of the rabbit prion protein, J. Biol. Chem., 2010, 285(41), 31682-31693.

11 R. Zahn, et al., NMR solution structure of the human prion protein, Proc. Natl. Acad. Sci. U. S. A., 2000, 97(1), 145-150.

12 M. Meli, M. Gasset and G. Colombo, Dynamic diagnosis of familial prion diseases supports the $\beta 2-\alpha 2$ loop as a universal interference target, PLoS One, 2011, 6(4), e19093.

$13 \mathrm{G}$. Ilc, et al., NMR structure of the human prion protein with the pathological Q212P mutation reveals unique structural features, PLoS One, 2010, 5(7), e11715.

14 D. B. O'sullivan, C. E. Jones, S. R. Abdelraheim, M. W. Brazier, H. Toms, D. R. Brown and J. H. Viles, Dynamics of a truncated prion protein, prp (113-231), from (15)N NMR relaxation: order parameters calculated and slow conformational fluctuations localized to a distinct region, Protein Sci., 2009, 18(2), 410-423.

15 L. Calzolai and R. Zahn, Influence of ph on NMR structure and stability of the human prion protein globular domain, J. Biol. Chem., 2003, 278(37), 35592-35596.

16 Y. Zhang, W. Swietnicki, M. G. Zagorski, W. K. Surewicz and F. D. Sönnichsen, Solution Structure of the E200K Variant of Human Prion Protein Implications for the mechanism of pathogenesis in familial prion diseases, J. Biol. Chem., 2000, 275(43), 33650-33654.

17 R. Diaz-Espinoza and C. Soto, High-resolution structure of infectious prion protein: the final frontier, Nat. Struct. Mol. Biol., 2012, 19(4), 370-377.

$18 \mathrm{H}$. Wille, et al., Structural studies of the scrapie prion protein by electron crystallography, Proc. Natl. Acad. Sci. U. S. A., 2002, 99(6), 3563-3568.

19 C. Govaerts, H. Wille, S. B. Prusiner and F. E. Cohen, Evidence for assembly of prions with left-handed $\beta$-helices into trimers, Proc. Natl. Acad. Sci. U. S. A., 2004, 101(22), 8342-8347.

20 M. L. Demarco and V. Daggett, From conversion to aggregation: protofibril formation of the prion protein, Proc. Natl. Acad. Sci. U. S. A., 2004, 101(8), 2293-2298.

21 N. J. Cobb, F. D. Sönnichsen, H. Mchaourab and W. K. Surewicz, Molecular architecture of human prion protein amyloid: a parallel, in-register $\beta$-structure, Proc. Natl. Acad. Sci. U. S. A., 2007, 104(48), 18946-18951.

22 N. Blinov, M. Berjanskii, D. S. Wishart and M. Stepanova, Structural domains and main-chain flexibility in prion proteins, Biochemistry, 2009, 48(7), 1488-1497.

23 B. Christen, S. Hornemann, F. F. Damberger and K. Wüthrich, Prion protein NMR structure from tammar wallaby (Macropus eugenii) shows that the $\beta 2-\alpha 2$ loop is modulated by long-range sequence effects, J. Mol. Biol., 2009, 389(5), 833-845.

24 S. Lee, L. Antony, R. Hartmann, K. J. Knaus, K. Surewicz, W. K. Surewicz and V. C. Yee, Conformational diversity in prion protein variants influences intermolecular $\beta$-sheet formation, $E M B O$ J. , 2010, 29(1), 251-262.

25 P. Derreumaux, Evidence that the 127-164 region of prion proteins has two equi-energetic conformations with $\beta$ or $\alpha$ features, Biophys. J., 2001, 81(3), 1657-1665.

26 G. Rossetti, et al., Structural facets of disease-linked human prion protein mutants: a molecular dynamic study, Proteins: Struct., Funct., Bioinf., 2010, 78(16), 3270-3280.

27 K. P. Santo, M. Berjanskii, D. S. Wishart and M. Stepanova, Comparative analysis of essential collective dynamics and NMR-derived flexibility profiles in evolutionarily diverse prion proteins, Prion, 2011, 5(3), 188-200.

$28 \mathrm{~J}$. Chen and D. Thirumalai, Helices 2 and 3 are the initiation sites in the prpc $\rightarrow$ prpsc transition, Biochemistry, 2012, 52(2), 310-319.

29 M. Adrover, et al., Prion fibrillization is mediated by a native structural element that comprises helices $\mathrm{H} 2$ and H3, J. Biol. Chem., 2010, 285(27), 21004-21012.

30 N. Chakroun, S. Prigent, C. A. Dreiss, S. Noinville, C. Chapuis, F. Fraternali and H. Rezaei, The 
oligomerization properties of prion protein are restricted to the H2H3 domain, FASEB J., 2010, 24(9), 3222-3231.

31 R. Tycko, R. Savtchenko, V. G. Ostapchenko, N. Makarava and I. V. Baskakov, The $\alpha$-helical C-terminal domain of full-length recombinant prp converts to an in-register parallel $\beta$-sheet structure in prp fibrils: evidence from solid state nuclear magnetic resonance, Biochemistry, 2010, 49(44), 9488-9497.

32 R. I. Dima and D. Thirumalai, Exploring the propensities of helices in prp $\mathrm{C}$ to form $\beta$ sheet using NMR structures and sequence alignments, Biophys. J., 2002, 83(3), 1268-1280.

33 G. Giachin, I. Biljan, G. Ilc, J. Plavec and G. Legname, Probing early misfolding events in prion protein mutants by NMR spectroscopy, Molecules, 2013, 18(8), 9451-9476.

34 E. Caldarulo, A. Barducci, K. Wüthrich and M. Parrinello, Prion protein $\beta 2-\alpha 2$ loop conformational landscape, Proc. Indian Natl. Sci. Acad., 2017, 114(36), 9617-9622.

35 M. W. Van der Kamp and V. Daggett, Pathogenic mutations in the hydrophobic core of the human prion protein can promote structural instability and misfolding, J. Mol. Biol., 2010, 404(4), 732-748.

$36 \mathrm{~J}$. Zuegg and J. E. Gready, Molecular dynamics simulations of human prion protein: importance of correct treatment of electrostatic interactions, Biochemistry, 1999, 38(42), 13862-13876.

37 R. Riek, G. Wider, M. Billeter, S. Hornemann, R. Glockshuber and K. Wüthrich, Prion protein NMR structure and familial human spongiform encephalopathies, Proc. Natl. Acad. Sci. U. S. A., 1998, 95(20), 11667-11672.

38 W. C. Guest, N. R. Cashman and S. S. Plotkin, Electrostatics in the stability and misfolding of the prion protein: salt bridges, self energy, and solvation, Int. J. Biochem. Cell Biol., 2010, 88(2), 371-381.

39 C. J. Cheng and V. Daggett, Molecular dynamics simulations capture the misfolding of the bovine prion protein at acidic ph, Biomolecules, 2014, 4(1), 181-201.

40 E. El-Bastawissy, M. H. Knaggs and I. H. Gilbert, Molecular dynamics simulations of wild-type and point mutation human prion protein at normal and elevated temperature, J. Mol. Graphics Modell., 2001, 20(2), 145-154.

41 S. Santini, J. B. Claude, S. Audic and P. Derreumaux, Impact of the tail and mutations G131V and M129V on prion protein flexibility, Proteins: Struct., Funct., Bioinf., 2003, 51(2), 258265.

42 J. Singh and J. B. Udgaonkar, Unraveling the molecular mechanism of ph-induced misfolding and oligomerization of the prion protein, J. Mol. Biol., 2016, 428(6), 1345-1355.

43 S. Capellari, P. Parchi, C. M. Russo, J. Sanford, M. S. Sy, P. Gambetti and R. B. Petersen, Effect of the E200K mutation on prion protein metabolism: comparative study of a cell model and human brain, Am. J. Pathol., 2000, 157(2), 613-622.

44 Y. Friedman-Levi, et al., Fatal prion disease in a mouse model of genetic E200K Creutzfeldt-Jakob disease, PLoS Pathog., 2011, 7(11), e1002350.
45 I. Biljan, G. Giachin, G. Ilc, I. Zhukov, J. Plavec and G. Legname, Structural basis for the protective effect of the human prion protein carrying the dominant-negative E219K polymorphism, Biochem. J., 2012, 446(2), 243-251.

46 S. Jahandideh, M. Jamalan and M. Faridounnia, Molecular dynamics study of the dominant-negative E219K polymorphism in human prion protein, J. Biomol. Struct. Dyn., 2015, 33(6), 1315-1325.

47 D. Shukla, C. X. Hernández, J. K. Weber and V. S. Pande, Markov state models provide insights into dynamic modulation of protein function, Acc. Chem. Res., 2015, 48(2), 414-422.

48 C. R. Schwantes, D. Shukla and V. S. Pande, Markov state models and tICA reveal a nonnative folding nucleus in simulations of NuG2, Biophys. J., 2016, 110(8), 1716-1719.

49 A. P. Collins and P. C. Anderson, Complete Coupled Binding-Folding Pathway of the Intrinsically Disordered Transcription Factor Protein Brinker Revealed by Molecular Dynamics Simulations and Markov State Modeling, Biochemistry, 2018, 57(30), 4404-4420.

50 B. E. Husic, R. T. McGibbon, M. M. Sultan and V. S. Pande, Optimized parameter selection reveals trends in Markov state models for protein folding, J. Chem. Phys., 2016, 145(19), 194103.

51 E. F. Pettersen, T. D. Goddard, C. C. Huang, G. S. Couch, D. M. Greenblatt, E. C. Meng and T. E. Ferrin, UCSF Chimera-a visualization system for exploratory research and analysis, J. Comput. Chem., 2004, 25(13), 1605-1612.

52 M. J. Abraham, T. Murtola, R. Schulz, S. Páll, J. C. Smith, B. Hess and E. Lindahl, GROMACS: high performance molecular simulations through multi-level parallelism from laptops to supercomputers, SoftwareX, 2015, 1, 19-25.

53 W. D. Cornell, et al., A second generation force field for the simulation of proteins, nucleic acids, and organic molecules, J. Am. Chem. Soc., 1996, 117, 5179-5197.

54 W. L. Jorgensen, J. Chandrasekhar, J. D. Madura, R. W. Impey and M. L. Klein, Comparison of simple potential functions for simulating liquid water, J. Chem. Phys., 1983, 79(2), 926-935.

55 G. Bussi, D. Donadio and M. Parrinello, Canonical sampling through velocity rescaling, J. Chem. Phys., 2007, 126(1), 014101.

56 H. J. Berendsen, J. V. Postma, W. F. van Gunsteren, A. R. H. J. Dinola and J. R. Haak, Molecular dynamics with coupling to an external bath, J. Chem. Phys., 1984, 81(8), 3684-3690.

57 U. Essmann, L. Perera, M. L. Berkowitz, T. Darden, H. Lee and L. G. Pedersen, A smooth particle mesh Ewald method, J. Chem. Phys., 1995, 103(19), 8577-8593.

58 B. Hess, H. Bekker, H. J. Berendsen and J. G. Fraaije, LINCS: a linear constraint solver for molecular simulations, $J$. Comput. Chem., 1997, 18(12), 1463-1472.

59 M. Parrinello and A. Rahman, Polymorphic transitions in single crystals: a new molecular dynamics method, J. Appl. Phys., 1981, 52(12), 7182-7190.

60 D. R. Roe and T. E. Cheatham III, PTRAJ and CPPTRAJ: software for processing and analysis of molecular 
dynamics trajectory data, J. Chem. Theory Comput., 2013, 9(7), 3084-3095.

61 W. Humphrey, A. Dalke and K. Schulten, VMD: visual molecular dynamics, J. Mol. Graphics, 1996, 14(1), 33-38.

62 J. H. Prinz, et al., Markov models of molecular kinetics: generation and validation, J. Chem. Phys., 2011, 134, 174105.

$63 \mathrm{~J}$. D. Chodera and F. Noé, Markov state models of biomolecular conformational dynamics, Curr. Opin. Struct. Biol., 2014, 25, 135-144.

64 J. D. Chodera, N. Singhal, V. S. Pande, K. A. Dill and W. C. Swope, Automatic discovery of metastable states for the construction of Markov models of macromolecular conformational dynamics, J. Chem. Phys., 2007, 126(15), $04 \mathrm{~B} 616$.

65 A. Sirur, D. De Sancho and R. B. Best, Markov state models of protein misfolding, J. Chem. Phys., 2016, 144(7), 075101.

66 M. K. Scherer, B. Trendelkamp-Schroer, F. Paul, G. PérezHernández, M. Hoffmann, N. Plattner, C. Wehmeyer, J. H. Prinz and F. Noé, Pyemma 2: A Software Package for Estimation, Validation, and Analysis of Markov Models, $J$. Chem. Theory Comput., 2015, 11(11), 5525-5542.

$67 \mathrm{H}$. Wu and F. Noé, Variational approach for learning Markov processes from time series data, arXiv:1707.04659, 2017.

68 F. Noé, C. Schütte, E. Vanden-Eijnden, L. Reich and T. R. Weikl, Constructing the equilibrium ensemble of folding pathways from short off-equilibrium simulations, Proc. Natl. Acad. Sci. U. S. A., 2009, 06(45), 19011-19016.

69 T. J. Dolinsky, P. Czodrowski, H. Li, J. E. Nielsen, J. H. Jensen, G. Klebe and N. A. Baker, PDB2PQR: expanding and upgrading automated preparation of biomolecular structures for molecular simulations, Nucleic Acids Res., 2007, 35(2), W522-W525.

70 N. A. Baker, D. Sept, S. Joseph, M. J Holst and J. A. Mccammon, Electrostatics of nanosystems: application to microtubules and the ribosome, Proc. Natl. Acad. Sci. U. S. A., 2001, 98(18), 10037-10041.

$71 \mathrm{H}$. Li, A. D. Robertson and J. H. Jensen, Very fast empirical prediction and rationalization of protein pka values, Proteins: Struct., Funct., Bioinf., 2005, 61(4), 704-721.
72 B. Han, Y. Liu, S. W. Ginzinger and D. S. Wishart, SHIFTX2: significantly improved protein chemical shift prediction, $J$. Biomol. NMR, 2011, 50(1), 43-57.

73 R Development Core Team, $R$ : A language and environment for statistical computing. $\mathrm{R}$ Foundation for Statistical Computing, Vienna, Austria, 2008, ISBN 3-900051-07-0, http://www.R-project.org.

74 F. Noé and S. Fischer, Transition networks for modeling the kinetics of conformational change in macromolecules, Curr. Opin. Struct. Biol., 2008, 18(2), 154-162.

75 G. Borgohain, N. Dan and S. Paul, Use of molecular dynamics simulation to explore structural facets of human prion protein with pathogenic mutations, Biophys. Chem., 2016, 213, 32-39.

76 Y. Chebaro and P. Derreumaux, The conversion of helix $\mathrm{H} 2$ to beta-sheet is accelerated in the monomer and dimer of the prion protein upon T183A mutation, J. Phys. Chem. B, 2009, 113(19), 6942-6948.

77 S. Zhou, D. Shi, X. Liu, H. Liu and X. Yao, Protective V127 prion variant prevents prion disease by interrupting the formation of dimer and fibril from molecular dynamics simulations, Sci. Rep., 2016, 24(6), 21804.

78 M. L. DeMarco and V. Daggett, Molecular mechanism for low ph triggered misfolding of the human prion protein, Biochemistry, 2007, 46(11), 3045-3054.

79 S. Menon and N. Sengupta, Perturbations In Inter-Domain Associations May Trigger The Onset Of Pathogenic Transformations In $\operatorname{Prp}(\mathrm{C})$ : Insights From Atomistic Simulations, Mol. BioSyst., 2015, 11(5), 1443-1453.

80 G. Rossetti, X. Cong, R. Caliandro, G. Legname and P. Carloni, Common structural traits across pathogenic mutants of the human prion protein and their implications for familial prion diseases, J. Mol. Biol., 2011, 411(3), 700-712.

81 G. Rossetti, G. Giachin, G. Legname and P. Carloni, Structural facets of disease-linked human prion protein mutants: a molecular dynamic study, Proteins: Struct., Funct., Bioinf., 2010, 78(16), 3270-3280. 